\title{
Internal Inductance and Conductor Loss Associated with the Ground Plane of a Microstrip Line
}

\author{
Christopher L. Holloway, Member, IEEE, and George A. Hufford, Life Fellow, IEEE
}

\begin{abstract}
In previous work, a closed-form expression for the current density on the ground plane of a microstrip line was presented. In this paper, we show how this formula for the current density is used to derive an expression for the internal inductance associated with the ground plane. Results are presented for different geometries that illustrate when the internal inductance of the ground becomes comparable to the external inductance of the microstrip line. We also illustrate that the integrals needed for the internal inductance calculation can be used to develop an expression for the conductor loss associated with the ground plane.
\end{abstract}

\section{INTRODUCTION}

$\mathbf{T}$ HE TOTAL inductance associated with the microstrip line shown in Fig. 1 is given by

$$
L_{T}=L_{e x}+L_{g r}+L_{\text {trace }}
$$

where $L_{e x}$ is the external inductance of the microstrip and is approximated by [1]

$$
L_{e x}=\frac{\mu_{o}}{4 \pi} \ln \left\{1+\frac{32 h^{2}}{w^{2}}\left[1+\sqrt{1+\left(\frac{\pi w}{8 h}\right)^{2}}\right]\right\} .
$$

$L_{g r}$ and $L_{\text {trace }}$ are the internal inductances of the ground plane and the trace, respectively. These two inductances result from the magnetic field penetration into the trace and ground conductors. This paper primarily concentrates on the ground plane inductance, with a brief discussion of the trace inductance in the conclusion.

The internal inductance of the ground plane is governed by the skin effect (i.e., the skin depth associated with the ground plane). As a result, it is known qualitatively that at high frequencies, the total inductance $L_{T}$ is dominated by the external inductance and the internal inductance can be neglected. However, for a given frequency there has not been a way of quantitatively determining how large or small the contribution of the internal ground inductance is compared to the total inductance.

In this paper, we introduce a closed-form expression for the internal inductance of the ground plane. With this expression, we can quantitatively determine what percentage of the total inductance is associated with the internal ground plane inductance for a given frequency.

Manuscript received July 2, 1996; revised January 21, 1997.

The authors are with the Institute for Telecommunication Sciences, United States Department of Commerce, Boulder, CO 80303 USA.

Publisher Item Identifier S 0018-9375(97)03698-3.

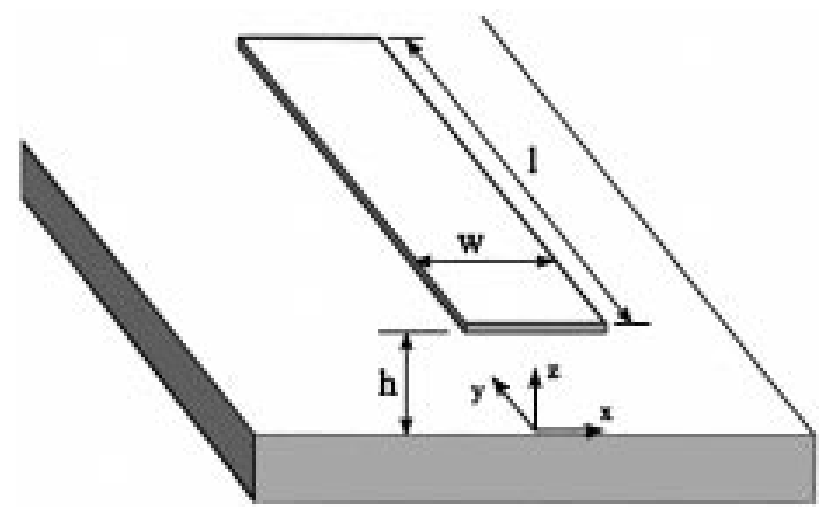

Fig. 1. Microstrip geometry.

\section{Ground Plane Inductance}

The internal inductance of the ground plane is given by [2]

$$
L_{g r}=\frac{1}{I^{2}} \int_{V} \bar{B} \cdot \bar{H} d v
$$

where $I$ is the total current flowing in the ground and $\bar{B}$ and $\bar{H}$ are the magnetic flux density and magnetic field intensity inside the ground plane, respectively. This integral is evaluated over the volume of the ground plane and the volume element $d v$ is expressed as $d v=d x d y d z$. For an infinitely long microstrip line, the fields are uniform in the $y$ direction. Thus, the integral along $d y$ for a given length $l$ of a microstrip line reduces to

$$
\int d y=l
$$

In general, the magnetic fields inside the ground plane have both an $x$ and $z$ component. However, in [3]-[5] it was shown that inside a highly conducting region the tangential component of the fields (the $x$ component in this case) dominates the normal component (the $z$ component), and to zeroth-order, $H_{z} \approx 0$. By utilizing this fact and assuming that the ground plane is nonmagnetic, (3) reduces to

$$
L_{g r}=\frac{\mu_{o} l}{I^{2}} \int_{-\infty}^{0} \int_{-\infty}^{\infty} H_{x}^{2} d x d z
$$

Also, in [3]-[5] it was shown that the fields exhibit an exponential decay of $e^{z / \delta}$ (where $\delta$ is the skin depth) away from the surface of a conducting region. Therefore, by assuming that the magnetic field inside the ground plane is governed by 
this same skin depth behavior, the field can be expressed as

$$
H_{x}=H_{o x}(x) e^{z / \delta}
$$

where $\delta$ is the skin depth of the ground plane and $H_{o x}(x)$ is the value of the magnetic field on the surface of the ground plane and is a function of $x$. For good conductors, the magnetic field on the surface of the ground is only slightly perturbed from that of the perfect conductor. Therefore, by using the boundary condition for the tangential component of the magnetic field on a perfect conductor, $H_{o x}(x)$ can be approximated by

$$
H_{o x}(x)=J_{g r}(x)
$$

where $J_{g r}(x)$ is the surface current density of the ground plane. Upon substituting (6) and (7) into (5), the $z$ integration can be evaluated explicitly and the internal inductance of the ground plane reduces to

$$
L_{g r}=\frac{\mu_{o} \delta l}{2} \int_{-\infty}^{\infty}\left[\frac{J_{g r}(x)}{I}\right]^{2} d x .
$$

If the current density on the ground plane is known, it can be substituted into (8) to obtain the internal inductance of the ground. In earlier work [6], the surface current density on a perfectly conducting ground plane was derived via a quasistatic Green's function approach and was shown to be

$$
J_{g r}(x)=\frac{I}{w \pi}\left[\tan ^{-1}\left(\frac{w-2 x}{2 h}\right)+\tan ^{-1}\left(\frac{w+2 x}{2 h}\right)\right]
$$

where $w$ and $h$ are defined in Fig. 1. While this expression is for a perfectly conducting ground plane, the actual current density on the surface of the highly conducting ground plane is only slightly perturbed from that of a perfect conductor. Therefore, the current density for a perfectly conducting ground plane can be used with little loss of generality. With this approximation, (8) reduces to

$$
\begin{aligned}
L_{g r}= & \frac{\mu_{o} \delta l}{2(w \pi)^{2}} \int_{-\infty}^{\infty}\left[\tan ^{-1}\left(\frac{w-2 x}{2 h}\right)\right. \\
& \left.+\tan ^{-1}\left(\frac{w+2 x}{2 h}\right)\right]^{2} d x .
\end{aligned}
$$

\section{A. Integral Evaluation}

The integral that we need to evaluate is rewritten as

$$
\mathcal{I}=\int_{-\infty}^{\infty}\left[\tan ^{-1}\left(\frac{x}{b}+a\right)-\tan ^{-1}\left(\frac{x}{b}-a\right)\right]^{2} d x
$$

where

and

$$
a=\frac{w}{2 h}
$$

$$
b=h .
$$

Through a change of variable where $\hat{x}=x / b,(11)$ is written as

$$
\mathcal{I}=b \int_{-\infty}^{\infty}\left[\tan ^{-1}(\hat{x}+a)-\tan ^{-1}(\hat{x}-a)\right]^{2} d \hat{x} .
$$

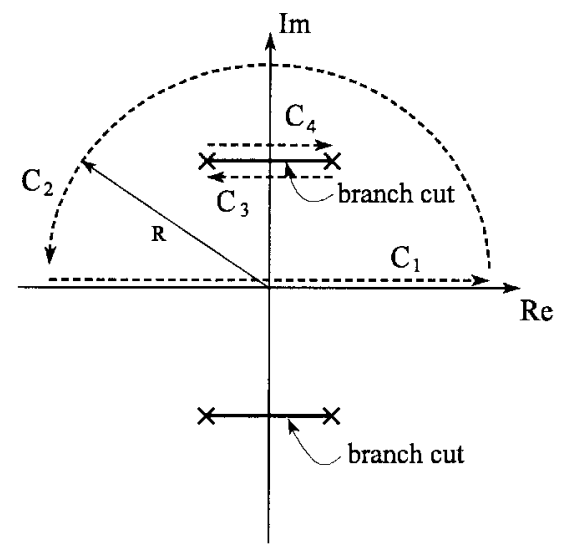

Fig. 2. Illustration of the contours needed for evaluating the integral.

Recall that $\tan ^{-1}$ can be expressed as $\ln$ by

$$
\tan ^{-1}(x)=\frac{1}{2 i} \ln \frac{i-x}{i+x} .
$$

The integral in (13) can now be expressed as

$$
\mathcal{I}=b \int_{-\infty}^{\infty}[f(\hat{x}, a)]^{2} d \hat{x}
$$

where

$$
f(\hat{x}, a)=\frac{1}{2 i}\left[\ln \left(\frac{i-a-\hat{x}}{i+a-\hat{x}}\right)+\ln \left(\frac{i-a+\hat{x}}{i+a+\hat{x}}\right)\right] .
$$

This function has four logarithmic singularities at $\hat{x}= \pm a \pm i$. For $\hat{x}$ near $\infty$, the rational function that is the argument for the logarithm equals $1+O\left(1 / x^{2}\right)$, hence $f=O\left(1 / x^{2}\right)$. Thus the integrand for $\mathcal{I}$ is $O\left(1 / x^{4}\right)$; this not only assures that the integral converges but also suggests that a contour integral with a large semicircle near infinity is useful. The singularities may be treated as two branch cuts, one joining the points $\pm a+i$ and the other joining $\pm a-i$ (Fig. 2). The arguments will increase or decrease by $2 \pi$ as one circles the points of one of these pairs. Since they appear as one dividing the other, these changes cancel out, giving a single-valued analytic function in the cut plane.

From complex analysis [7], this integral can be evaluated by choosing the contour shown in Fig. 2:

$$
\int_{C}=\int_{C_{1}}+\int_{C_{2}}+\int_{C_{3}}+\int_{C_{4}} .
$$

Since this contour does not capture any poles, the Cauchy Integral Theorem states that $\int_{C}=0$. As $R$ approaches infinity, the contribution from the contour $C_{2}$ goes to zero and contour $C_{1}$ reduces to an integral along the entire real axis $(-\infty, \infty)$. Therefore, the integral along the real axis can be written as the sum of the two integrals around the branch cuts $C_{3}$ and $C_{4}$

$$
\begin{aligned}
\int_{-\infty}^{\infty}[f(\hat{x}, a)]^{2} d \hat{x}= & \int_{-a}^{a}\left[f_{-}(\hat{x}+i, a)\right]^{2} d \hat{x} \\
& -\int_{-a}^{a}\left[f_{+}(\hat{x}+i, a)\right]^{2} d \hat{x}
\end{aligned}
$$

where $f_{-}(\hat{x}+i, a)$ and $f_{+}(\hat{x}+i, a)$ correspond to the function on the lower and upper side of the branch cut. Only the first 
logarithm of (16) changes values across the branch cut. Thus, $f_{-}(\hat{x}+i, a)$ and $f_{+}(\hat{x}+i, a)$ are given by

$$
f_{-}(\hat{x}+i, a)=\frac{1}{2 i}\left[\ln \left|\frac{a+\hat{x}}{a-\hat{x}}\right|+\ln \frac{2 i-a+\hat{x}}{2 i+a+\hat{x}}\right]+\frac{\pi}{2}
$$

and

$$
f_{+}(\hat{x}+i, a)=\frac{1}{2 i}\left[\ln \left|\frac{a+\hat{x}}{a-\hat{x}}\right|+\ln \frac{2 i-a+x}{2 i+a+x}\right]-\frac{\pi}{2} .
$$

Substituting these two expressions into (13) and (18) leads to

$$
\mathcal{I}=\frac{b \pi}{i} \int_{-a}^{a}\left[\ln \left|\frac{a+\hat{x}}{a-\hat{x}}\right|+\ln \frac{2 i-a+\hat{x}}{2 i+a+\hat{x}}\right] d \hat{x} .
$$

The first logarithm is an odd function of $\hat{x}$ so that its integral vanishes. The integral of the second logarithm is elementary. Thus

$$
\mathcal{I}=4 \pi a b \tan ^{-1}(a)-2 \pi b \ln \left(1+a^{2}\right) .
$$

With this expression for $\mathcal{I}$ and the use of (12) the internal inductance per unit length of the ground plane is given by

$$
\begin{aligned}
\frac{L_{g r}}{l} & =\frac{\mu_{o} \delta}{w \pi}\left\{\tan ^{-1}\left(\frac{w}{2 h}\right)-\frac{h}{w} \ln \left[1+\left(\frac{w}{2 h}\right)^{2}\right]\right\} \\
\text { or } & \frac{L_{g r}}{l}=\frac{2}{w \pi} \frac{R_{s}}{\omega}\left\{\tan ^{-1}\left(\frac{w}{2 h}\right)-\frac{h}{w} \ln \left[1+\left(\frac{w}{2 h}\right)^{2}\right]\right\}
\end{aligned}
$$

where $R_{s}$ is the Leontovich surface impedance [8] and is given by

$$
R_{s}=\frac{1}{\sigma \delta}
$$

where $\sigma$ is the conductivity of the ground plane. While (23)-(25) are only valid for an infinitely thick ground plane, a discussion on their use for thin ground planes is given in Section VI.

\section{RESUlts FOR TyPICAL GeOMETRIES}

The relative importance of $L_{g r}$ as compared to $L_{T}$ is a function of frequency and the microstrip geometry. In this section, four different geometries are analyzed: two sets of geometries used commonly in microwave and millimeter-wave integrated circuits (MIMIC's), and two sets of geometries used commonly in printed circuit boards.

The first geometry is a microstrip on GaAs substrate with $h=100 \mu \mathrm{m}, w=70 \mu \mathrm{m}, \sigma=5.8 \cdot 10^{7}$, and $\epsilon_{r}=12.9$. This corresponds to a line with a characteristic impedance of $51 \Omega$. Fig. 3 shows the results of the total inductance $\left(L_{T}=L_{e x}+L_{g r}\right)$, the external inductance $L_{e x}$, and the internal ground plane inductance $L_{g r}$. The internal ground plane inductance is small compared to the external inductance at high frequencies, but at the low frequencies the internal inductance is comparable to the external inductance. This is further illustrated in Fig. 4. In Fig. 4, the percent of internal inductance compared to total inductance $L_{T}$ is plotted as a function of frequency. For this figure, $\Delta L$ is defined as

$$
\Delta L=\frac{L_{g r}}{L_{e x}+L_{g r}} \times 100 .
$$

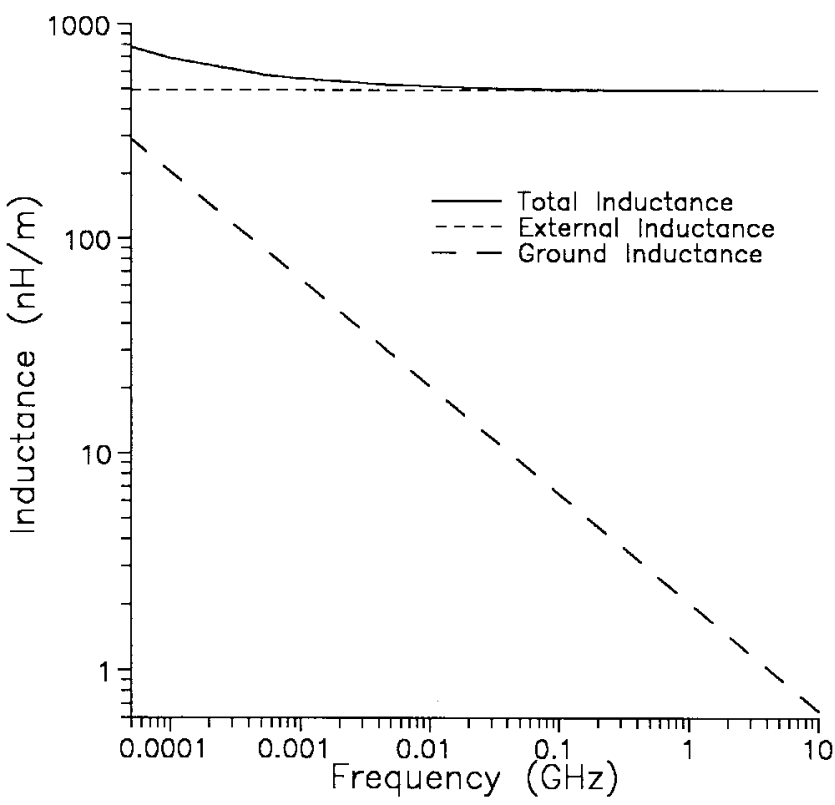

Fig. 3. Total, external, and internal inductances for a microstrip line with $w=70 \mu \mathrm{m}, h=100 \mu \mathrm{m}, \sigma=5.8 \cdot 10^{7}$, and $\epsilon_{r}=12.9\left(Z_{o}=51 \Omega\right)$.

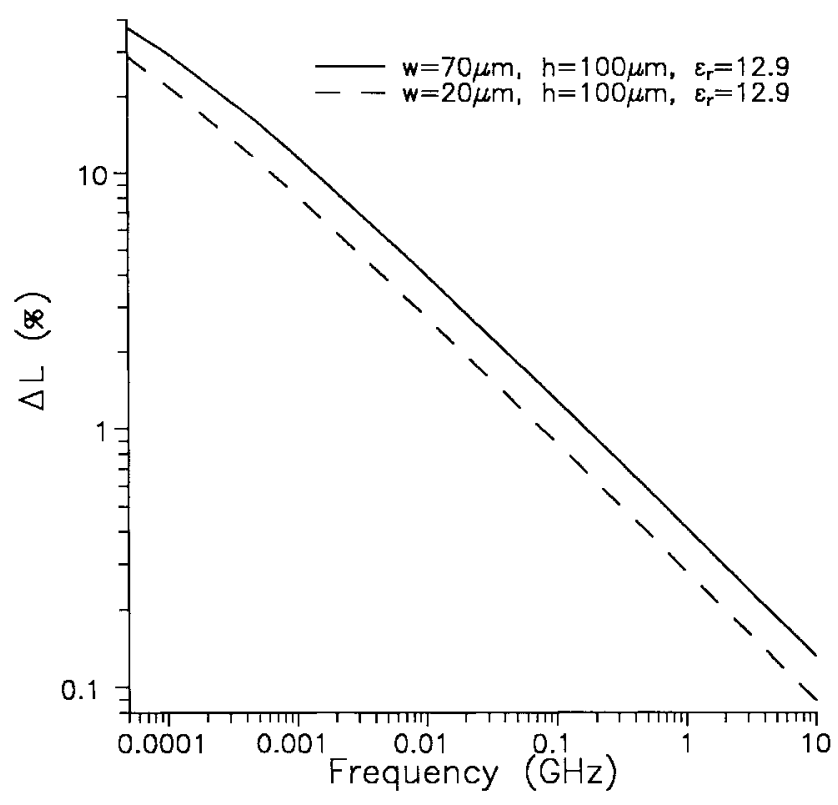

Fig. 4. The percent of internal inductance compared to total inductance $(\Delta L)$ for a $Z_{o}=51 \Omega$ and $Z_{o}=79 \Omega$ microstrip line with $\sigma=5.8 \cdot 10^{7}$.

Also shown in Fig. 4 are results for a $79 \Omega$ line, where $h=$ $100 \mu \mathrm{m}, w=20 \mu \mathrm{m}, \epsilon_{r}=12.9$, and $\sigma=5.8 \cdot 10^{7}$.

The last two examples correspond to microstrip lines fabricated on FR-4 $\left(\epsilon_{r}=4.5\right)$. The first case is a microstrip line with $h=0.1778 \mathrm{~mm}$ (7 mils),$w=0.381 \mathrm{~mm}(15 \mathrm{mils})$, $\sigma=5.8 \cdot 10^{7}$, and $\epsilon_{r}=4.5$. This corresponds to a line with a characteristic impedance of $46 \Omega$. Fig. 5 shows the results of the total inductance $\left(L_{T}=L_{e x}+L_{g r}\right)$, the external inductance $L_{e x}$, and the internal ground plane inductance $L_{g r}$. In Fig. 6 , the percent $\Delta L$ of internal inductance compared to total inductance $L_{T}$ is plotted as a function of frequency. Also 


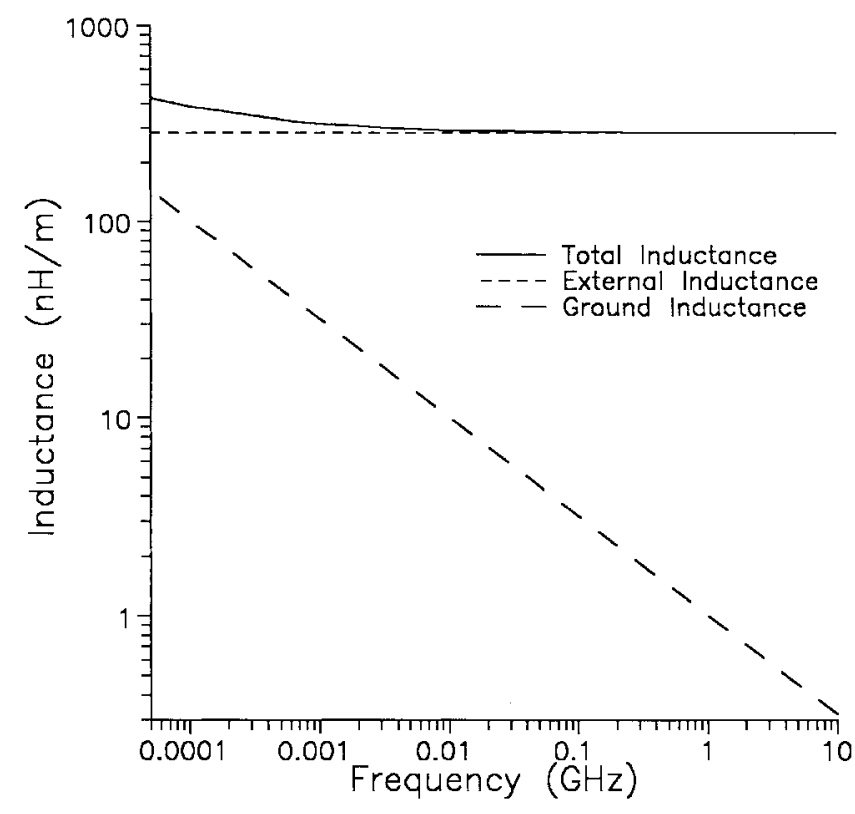

Fig. 5. Total, external, and internal inductances for a microstrip line with $w=381 \mu \mathrm{m}, h=178 \mu \mathrm{m}, \sigma=5.8 \cdot 10^{7}$, and $\epsilon_{r}=4.5\left(Z_{o}=46 \Omega\right)$.

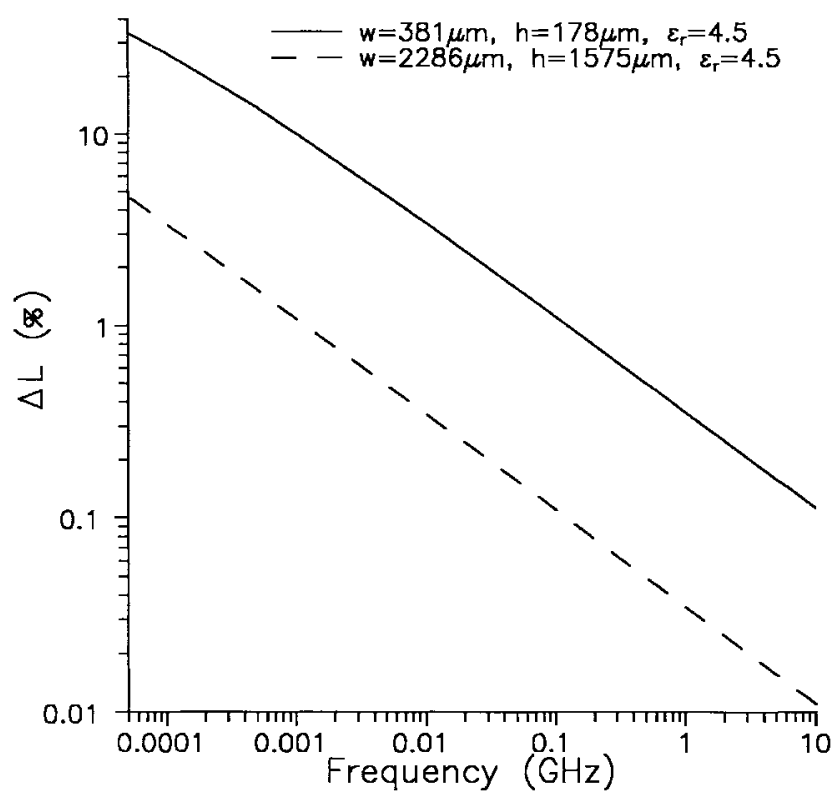

Fig. 6. The percent of internal inductance compared to total inductance $(\Delta L)$ for a $Z_{o}=46 \Omega$ and $Z_{o}=58 \Omega$ microstrip line with $\sigma=5.8 \cdot 10^{7}$.

shown in Fig. 6 are results for a $58 \Omega$ line, where $h=1.575$ $\mathrm{mm}$ (62 mils), $w=2.286 \mathrm{~mm}$ (90 mils), $\epsilon_{r}=4.5$, and $\sigma=$ $5.8 \cdot 10^{7}$. For high frequencies, the inductance of the ground is negligible, but becomes important at lower frequencies.

\section{GRound Plane Loss}

Using a standard wall loss perturbation analysis, the loss associated with the ground plane is given by [6]

$$
\alpha_{g r}=\frac{R_{s}}{2 Z_{o} I^{2}} \int_{-\infty}^{\infty}\left|J_{g r}(x)\right|^{2} d x .
$$

In [6] it was stated that this integral could not be evaluated explicitly, and numerical results were presented. However, this integral is identical to the one evaluated in Section II. With (11) and (22), the loss associated with the ground plane is given by

$$
\alpha_{g r}=\frac{R_{s}}{Z_{o} w \pi}\left\{\tan ^{-1}\left(\frac{w}{2 h}\right)-\frac{h}{w} \ln \left[1+\left(\frac{w}{2 h}\right)^{2}\right]\right\} .
$$

Results from this expression give the identical results that were presented in [6] which were obtained by numerically evaluating (27).

\section{CHARACTERISTIC IMPEDANCE}

The characteristic impedance is perturbed by the presence of the magnetic fields that penetrate the conductor. Inside the conductor the electric fields are zero to zeroth-order and the dominant fields in the conductor are magnetic [9]. The presence of the nonperfect conductor will only change the inductance of the line, and the capacitance $C$ is unchanged from that of a perfect conductor. Thus, the characteristic impedance is defined as

$$
\begin{aligned}
\hat{Z}_{c} & =\sqrt{\frac{R+j \omega L_{T}}{j \omega C}} \\
& =\sqrt{\frac{R+j \omega\left(L_{e x}+L_{g r}\right)}{j \omega C}} \\
& =\sqrt{Z_{o}^{2}+\frac{L_{g r}}{C}+\frac{R}{j \omega C}}
\end{aligned}
$$

where $Z_{o}=\sqrt{L_{e x} / C}$ is the characteristic impedance for perfect conductors, $R$ is the resistance due to the field penetration into the ground plane and is approximated by

$$
R \approx 2 Z_{o} \alpha_{g r}
$$

and $C$ is the capacitance of a microstrip with perfect conductors, and is approximated with an accuracy of $2 \%$ or better by [10]

$$
C \simeq \frac{2 \pi \epsilon_{o}\left(\epsilon_{r}+1\right)}{\ln \left(1+\frac{32 h}{w}\left\{\frac{h}{w} r_{\epsilon}+\sqrt{\left(\frac{h r_{\epsilon}}{w}\right)^{2}+\left[\frac{\pi\left(\epsilon_{r}+1\right)}{16 \epsilon_{r}}\right]^{2}}\right\}\right)}
$$

where

$$
r_{\epsilon} \simeq \sqrt{0.4052+\frac{0.516}{\epsilon_{r}}+\frac{0.0788}{\epsilon_{r}^{2}}}
$$

The losses in the ground plane result in a complex characteristic impedance having both a magnitude and phase. The percentage increase of the magnitude of the characteristic impedance $\Delta Z$ due to the fields penetrating into the ground 


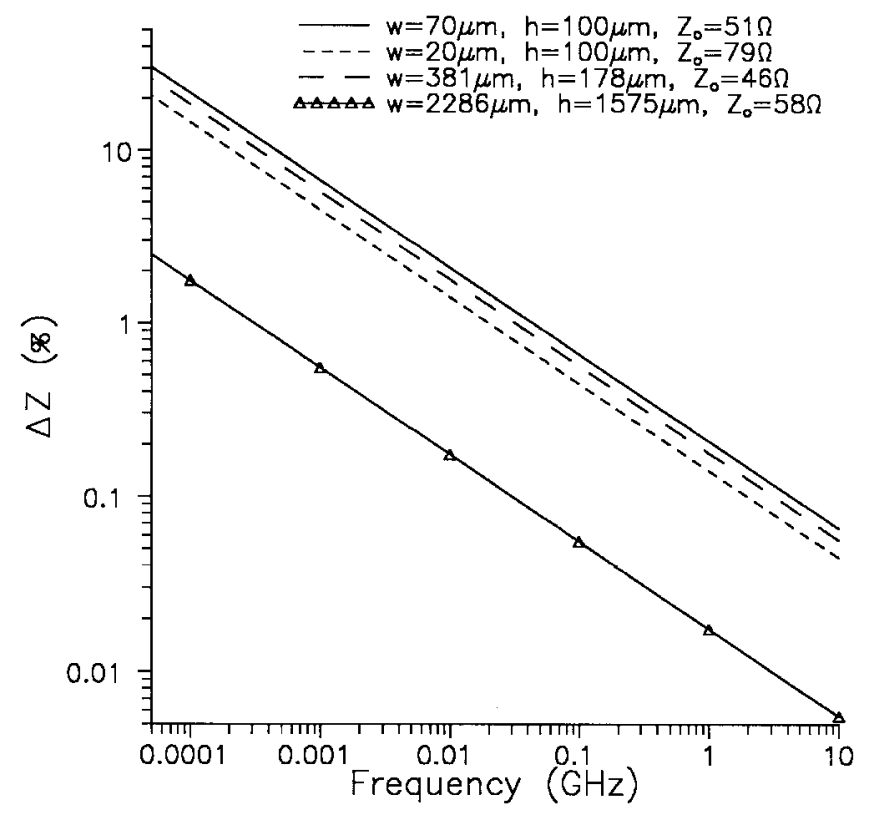

Fig. 7. The percent increase in the characteristic impedance due to field penetration into the ground plane.

plane is defined by

$$
\Delta Z=\frac{\left|\hat{Z}_{c}\right|-Z_{o}}{Z_{o}} \times 100 .
$$

Values for $\Delta Z$ for the four geometries analyzed above are shown in Fig. 7. These results illustrate how the characteristic impedance $\hat{Z}_{c}$ changes as the magnetic fields penetrate into the ground plane.

\section{SURFACE IMPEDANCE FOR THIN CONDUCTORS}

The derivation of both the internal inductance $L_{g r}$ and conductor loss $\alpha_{g r}$ of the ground plane assumed that the ground plane thickness $t$ was much larger than the skin depth $\delta$. This assumption allows for both the inductance and loss to be expressed in turns of the Leontovich surface impedance $R_{s}$ (which itself is only valid for $t \gg \delta$ ). In this section, the validity of the Leontovich surface impedance (and hence the validity of $L_{g r}$ and $\alpha_{g r}$ ) for a thin ground plane is discussed.

A generalized surface impedance boundary condition for a thin conductor was derived in [9] and [11]. Using those results, it can be shown that to zeroth-order, a thickness correction to the Leontovich surface impedance can be given by

$$
R_{t} \sim R_{s} \operatorname{Re}\left\{(1+j) \operatorname{coth}\left[(1+j) \frac{t}{\delta}\right]\right\} .
$$

This correction was also suggested by Lee and Itoh [12].

The surface impedance given in (34) reduces to the Leontovich surface impedance $R_{s}$ for $t / \delta \gg 1$. For $t / \delta=1$, there is an $8 \%$ difference in the surface impedances given in (25) and (34). The difference reduces to only $1 \%$ for $t / \delta=2.5$, and for $t / \delta>4$ there is at most only a $0.01 \%$ difference in the two expressions. Thus, the results presented here can be used for ground planes with thickness on the same order as the skin depth without significant loss in accuracy.

\section{CONCLUSION}

In this paper, we introduced a closed-form expression for the internal inductance of the ground plane associated with a microstrip line. This expression can be used to determine the internal ground plane inductance for any given frequency and geometry. The results presented here illustrate that the geometry of the line as well as the frequency play a strong role in determining the relative importance of the internal inductance. A closed-form expression for the conductor loss associated with the ground plane was also presented.

The effect of magnetic field penetration inside the ground plane on the characteristic impedance was investigated. It was shown that depending on the microstrip geometry, large changes in the characteristic impedance can occur at low frequencies due to this effect.

This paper deals exclusively with the effect of the change in the total inductance and the characteristic impedance associated with the ground plane. This is only part of the whole picture. The effect of magnetic fields penetrating inside the trace conductor also changes these two quantities. In [9], it was shown that for narrow trace widths, the loss in the trace was the dominant loss. Therefore, for narrow trace widths, the changes in total inductance and characteristic impedance may also be dominated by the trace conductor. An analysis of how these quantities change due to the trace conductor is more involved and will be investigated in the future.

\section{REFERENCES}

[1] H. A. Wheeler, "Transmission-line properties of a strip on a dielectric sheet on a plane," IEEE Trans. Microwave Theory Tech., vol. 25, pp. 631-647, 1977.

[2] C. T. A. Johnk, Engineering Electromagnetic Fields and Waves. New York: Wiley, 1975, ch. 5.

[3] S. M. Rytov, "Calcul du skin-effect par la mèthode des perturbations," J. Phys. (USSR), vol. 2, no. 3, pp. 233-242, 1940.

[4] M. Artola and M. Cessenat, "Diffraction d'une onde électromagnétique par un obstacle borné à permittivité et perméabilité élevées," C.R. Acd. Sci. Paris, t. 314, série 1, pp. 349-354, 1992.

[5] T. B. A. Senior, "Impedance boundary conditions for imperfectly conducting surfaces," Appl. Sci. Res., vol. 8, no. B8, pp. 418-436, 1960.

[6] C. L. Holloway and E. F. Kuester, "Closed-form expressions for the current density on the ground plane of a microstrip line, with application to ground plane loss," IEEE Trans. Microwave Theory Tech., vol. 43, pp. 1204-1207, 1995.

[7] W. R. LePage, Complex Variables and LaPlace Transform for Engineers. New York: McGraw-Hill, 1961, ch. 6.

[8] M. A. Leontovich, "Approximate boundary conditions for an electromagnetic fields at the surface of a highly conducting body," in Issledovaniya po Rasprostraneniyu Radiovoln (Research in Radio Wave Propagation, in Russian), Moscow, Russia: 1948, pt. 2, pp. 5-12; also M. A. Leontovich, in Izbrannye Trudy: Teoreticheskaya Fizika (A Collection of Work: Theoretical Physics). Moscow, Russia: 1985, pp. 351-355.

[9] C. L. Holloway and E. F. Kuester, "Edge shape effects and quasiclosed form expressions for the conductor loss of microstrip lines," Radio Sci., vol. 29, no. 3, pp. 539-559, 1994.

[10] E. F. Kuester, "Accurate approximations for a function appearing in the analysis of microstrip," IEEE Trans. Microwave Theory Tech., vol. 32, pp. 131-133, 1984.

[11] R. Horton, B. Easter, and A. Gopinath, "Variation of microstrip losses with thickness of strip," Electron. Lett., vol. 7, no. 17, pp. 490-491, 1971.

[12] H. Y. Lee and T. Itoh, "Phenomenological loss equivalence method for planar quasi-TEM transmission lines with a thin normal or superconductor," IEEE Trans. Microwave Theory Tech., vol. 37, pp. 1904-1909, 1989. 
Christopher L. Holloway (S'86-M'92) was born in Chattanooga, TN, on March 26, 1962. He received the B.S. degree from the University of Tennessee at Chattanooga in 1986, and the M.S. and Ph.D. degrees from the University of Colorado at Boulder, in 1988 and 1992, respectively, both in electrical engineering.

During 1992, he was a Research Scientist with Electro Magnetic Applications, Inc., Lakewood, CO. His responsibilities included theoretical analysis and finite-difference time-domain modeling of various electromagnetic problems. From the fall of 1992 to 1994, he was with the National Center for Atmospheric Research (NCAR), Boulder. While at NCAR his duties included wave propagation modeling, signal processing studies, and radar systems design. Since 1994, he has been with the Institute for Telecommunication Sciences (ITS), U.S. Department of Commerce, Boulder, where he is involved in wave propagation studies. His research interests include electromagnetic field theory, wave propagation, guided wave structures, remote sensing, numerical methods, and EMC/EMI issues.

Dr. Holloway is a member of Commission A of the International Union of Radio Science and an Associate Editor on propagation for the IEEE Transactions on Electromagnetic Compatibility.
George A. Hufford (S'45-A'49-M'55-LF'95) was born in San Francisco, CA, in 1927. He received the B.S. degree in engineering from the California Institute of Technology, Pasadena, in 1946, the M.S. degree in electrical engineering from the University of Washington, Seattle, in 1948, and the Ph.D. degree in mathematics from Princeton University, Princeton, NJ, in 1953.

In 1964, he joined the U.S. Department of Commerce as a member of the Tropospheric Telecommunications Laboratory, CRPL, Boulder, CO, and has remained there although the organization has undergone several name changes and is now the Spectrum Division of ITS, NTIA, in the Department of Commerce. In these years, he has been active in research related to the computation and modeling of tropospheric radio propagation under real-world conditions as when irregular terrain and a changing atmosphere are involved.

Dr. Hufford is a member of the American Mathematical Society, the Society of Industrial and Applied Mathematics, and Sigma Xi. 